\title{
Vegan Diet
}

National Cancer Institute

\section{Source}

National Cancer Institute. Vegan Diet. NCI Thesaurus. Code C15630.

A type of vegetarian diet that excludes all animal products, including dairy, eggs, meat, poultry, fish, and animal fats. 